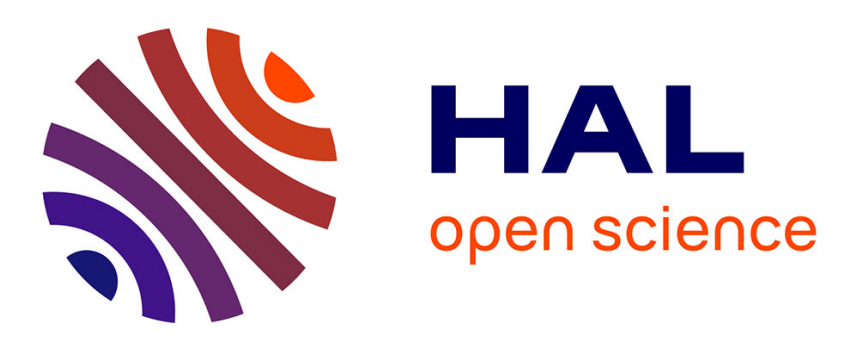

\title{
Modélisation des machines cylindriques asynchrones sans fer. Prise en compte de la longueur finie du rotor
}

\author{
F. Rioux-Damidau
}

\section{To cite this version:}

F. Rioux-Damidau. Modélisation des machines cylindriques asynchrones sans fer. Prise en compte de la longueur finie du rotor. Revue de Physique Appliquée, 1985, 20 (4), pp.235-242. 10.1051/rphysap:01985002004023500 . jpa-00245328

\section{HAL Id: jpa-00245328 https://hal.science/jpa-00245328}

Submitted on 1 Jan 1985

HAL is a multi-disciplinary open access archive for the deposit and dissemination of scientific research documents, whether they are published or not. The documents may come from teaching and research institutions in France or abroad, or from public or private research centers.
L'archive ouverte pluridisciplinaire HAL, est destinée au dépôt et à la diffusion de documents scientifiques de niveau recherche, publiés ou non, émanant des établissements d'enseignement et de recherche français ou étrangers, des laboratoires publics ou privés. 


\title{
Modélisation des machines cylindriques asynchrones sans fer. Prise en compte de la longueur finie du rotor
}

\author{
F. Rioux-Damidau \\ Laboratoire d'Electrotechnique des Universités Paris VI et XI (*), Bâtiment 214, 91405 Orsay, France
}

(Reçu le 21 mars 1984, révisé le 4 décembre 1984, accepté le 7 janvier 1985)

\begin{abstract}
Résumé. - Une précédente modélisation à base de séries de Fourier spatio-temporelles avait décrit le système formé du bobinage réel et d'une ceinture et d'un rotor infiniment longs. On tient compte ici de la longueur finie du rotor en effectuant des itérations où l'on soustrait les effets des courants induits dans la partie inexistante du rotor infini.
\end{abstract}

Abstract. - A previous modelling using a double space associated with a temporal Fourier analysis described the device constituted of the real winding and of a belt and a rotor of infinite lengths. The finite length of the rotor is taken into account by doing iterations where one subtracts the effects of the induced currents appearing in the non-existent part of the infinite rotor.

\section{Introduction.}

La structure des machines cylindriques asynchrones sans fer est très simple [2] : un cylindre métallique passif constituant le rotor est entouré d'un bobinage multipolaire formé uniquement de cuivre, l'ensemble étant logé dans une culasse en fer. Diverses modélisations successives, approchant de plus en plus le problème ont été effectuées [3,4]. La dernière et la plus précise est de nature semi-tridimensionnelle [1] : elle suppose que le bobinage réel est enserré entre un rotor et une ceinture très longs. La confrontation de cette modélisation avec l'expérience $[5,6]$ a montré sa validité lorsque la machine était «longue ", c'est-àdire lorsque sa longueur était très supérieure à son diamètre. Cela était prévisible car, au niveau des extrémités du bobinage de forme hélicoïdale [1], les conducteurs aller et retour sont très proches et créent des champs magnétiques très faibles. Une erreur sur la détermination de ces derniers se répercute très peu sur la valeur des performances globales. Lorsqu'au contraire la machine est courte, les conducteurs aller et retour se rejoignent sous un angle très ouvert; le champ au niveau des extrémités n'est plus négligeable et il devient nécessaire de le déterminer avec précision. Pour cela il faut tenir compte des longueurs exactes du rotor et de la ceinture. Cela est possible par la méthode des éléments finis, mais il est alors nécessaire de disposer d'un ordinateur très puissant [7].

(*) Equipe associée au C.N.R.S. no 838.
Nous présentons ici une étude qui tient compte de la longueur finie du rotor (mais qui suppose toujours la ceinture longue) et qui requiert un temps de calcul très court. Elle complète donc la modélisation présentée en [1], laquelle sera supposée connue.

\section{Méthode de calcul.}

Dans la modélisation semi-tridimensionnelle, nous avions considéré un système formé d'un rotor et d'une ceinture infiniment longs et de stators placés régulièrement autour du rotor (cf. Fig. 1); deux stators contigus sont symétriques l'un de l'autre. Pour déterminer les solutions des équations de Maxwell, nous décomposons toutes les grandeurs en séries de Fourier à la fois spatiales (en $\theta$ et $z$ ) et temporelles :

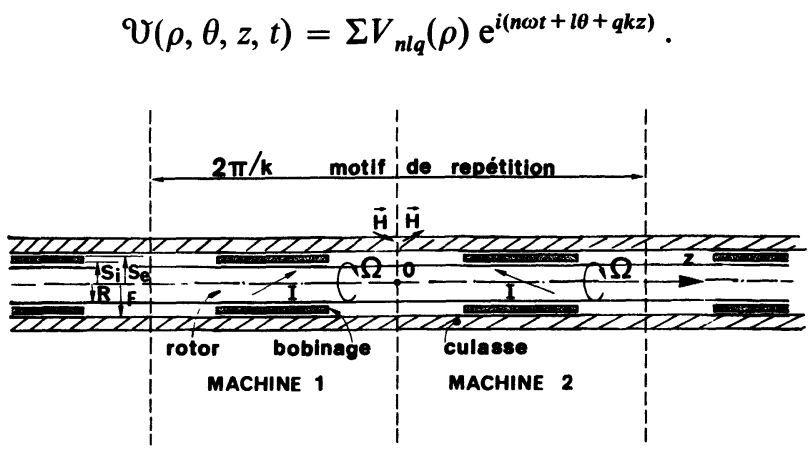

Fig. 1. - Le modèle avec rotor et ceinture infinis.

[The model with a rotor and a belt of infinite lengths.] 
Nous avions montré que les différents modes $n l q$ évoluent indépendamment les uns des autres. Autrement dit, le champ $\mathbf{H}_{n l q}(\rho)$ ne dépend que de $\mathbf{I}_{n l q}(\rho)$ (mêmes $n, l$ et $q$ ). Cette propriété est liée au modèle choisi : rotor et ceinture longs. En effet, dans ce cas, une grandeur telle que la conductivité du rotor est indépendante de $z$ et l'on peut écrire, pour $\rho<R$ :

$$
\nabla \wedge\left(\frac{\mathfrak{J}}{\sigma}\right)=\frac{1}{\sigma}(\nabla \wedge \mathfrak{J}),
$$

(cf. (15) de [1]), J étant le courant dans le rotor.

$\mathrm{Si}$ le rotor est de longueur finie, (de longueur supérieure, inférieure ou égale à celle du stator), la relation ci-dessus n'est plus valable et le problème se complique. On a :

$$
\nabla \wedge\left(\frac{\mathfrak{J}}{\sigma}\right)=\nabla\left(\frac{1}{\sigma}\right) \wedge \mathfrak{J}+\frac{1}{\sigma} \nabla \wedge \mathfrak{J}
$$

$\nabla\left(\frac{1}{\sigma}\right)$ étant proportionnel à la distribution de Dirac $\delta$ au niveau des extrémités du rotor et nul ailleurs. La composante de Fourier d'indices $n l q$ de $\nabla \wedge \mathfrak{J}$ ne fait intervenir que $I_{n l q}$ mais celle de $\nabla\left(\frac{1}{\sigma}\right) \wedge \mathfrak{J}$ fait intervenir tous les $I_{n l q^{\prime}}$ avec $q^{\prime}$ quelconque. Les équations vont donc mélanger les harmoniques entre eux, ce qui complique fortement les calculs. Pour éviter ce problème, nous avons préféré partir du modèle semi-tridimensionnel et lui appliquer des corrections successives.

Dans ce modèle, le rotor " infini " (c'est-à-dire en fait, le rotor de longueur $\pi / k$, cf. Fig. 1) est le siège de courants induits $\mathfrak{J}_{i}$ que nous sommes capables de calculer. Nous pouvons alors imposer artificiellement des courants sources $\boldsymbol{S}=-\mathfrak{J}_{\mathbf{i}}$ dans la partie du rotor infini qui, en réalité, n'existe pas (cf. Fig. 2) puis calculer l'effet de ces courants sources $\boldsymbol{\delta}$ sur le rotor infini. Il y a ainsi apparition de courants induits $\mathcal{N}$, dus à $\boldsymbol{S}$, dans le rotor infini que l'on va pouvoir calculer comme précédemment. Autrement dit, les courants imposés $\mathfrak{J}_{\mathrm{s}}$ dans le stator créent des courants $\mathfrak{J}_{\mathrm{i}}$ dans le rotor puis les courants sources $\mathbf{8}$

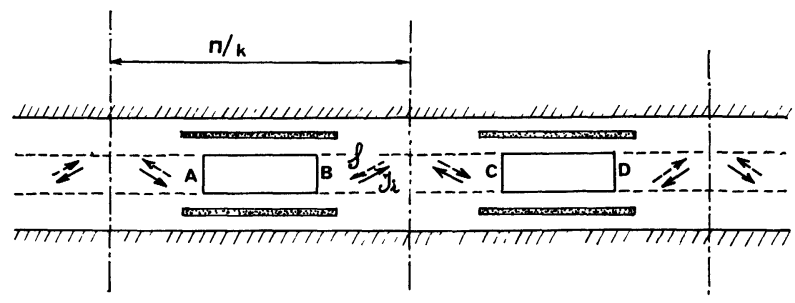

Fig. 2. - Les courants sources $\boldsymbol{S}$ sont opposés aux courants induits $\boldsymbol{J}_{\mathrm{i}}$ dans la région où n'existe que le rotor infini et pas le rotor réel.

[The source currents $\boldsymbol{S}$ are opposite to the induced currents $\mathfrak{J}_{i}$ in the region where is present solely the infinite rotor and not the real one.] (imposés quand on effectue le calcul) créent des courants induits $\mathcal{N}$, et bien sûr les courants induits $\mathcal{N}$ permettent de définir des courants sources $\boldsymbol{S}^{\prime}$ qui induisent des courants $\boldsymbol{N}^{\prime}$, etc. Si le calcul converge, on aura $S>S^{\prime}>S^{\prime \prime}$ etc. En superposant tous les ensembles $\left(\mathfrak{J}_{1}, \mathfrak{J}_{\mathfrak{i}}\right),(\boldsymbol{S}, \mathcal{N}),\left(\boldsymbol{S}^{\prime}, \mathcal{N}^{\prime}\right), \ldots$ on obtiendra un système avec un courant $\boldsymbol{J}_{\mathrm{s}}$ dans le stator et du courant induit seulement à l'endroit du rotor réel. On aura donc une description correcte du système réel.

Remarquons que les courants $\boldsymbol{S}$ ne se referment pas; ils représentent une tranche des courants découpée dans le rotor infini. Ils n'obéissent donc pas à $\boldsymbol{\nabla} . \boldsymbol{S}=\mathbf{0}$. Ce n'est que l'ensemble $\boldsymbol{N}+\boldsymbol{S}$, qui permet de calculer le champ magnétique par

$$
\nabla \wedge \mathfrak{H}=\boldsymbol{N}+\boldsymbol{S},
$$

qui satisfait à la divergence nulle : $\boldsymbol{\nabla} \cdot(\boldsymbol{N}+\boldsymbol{S})=0$. $\mathcal{N}$ étant le courant induit dans le rotor infini, il obéit à la loi d'Ohm :

$$
\mathcal{N}=\sigma\left(\boldsymbol{E}+\mathbf{v} \wedge \mu_{0} \mathfrak{H}\right) .
$$

Le calcul complet comporte deux étapes indépendantes que nous exposerons successivement. L'une est l'étude du système semi-tridimensionnel avec courants sources imposés. Ces courants sont soit dans le stator, soit dans le rotor. On déterminera, entre autres choses, le courant induit dans le rotor infini. L'autre effectue le calcul des courants sources $\mathbf{S}$ à partir des courants induits.

\section{Etude du système semi-tridimensionnel.}

Dans [1], nous avions effectué cette étude en prenant comme courants sources les courants statoriques. Nous prenons maintenant comme courants sources les courants $\boldsymbol{\delta}$ localisés dans le rotor et nous les supposons pour le moment connus. Nous écrivons les équations relatives au mode $n l q$ et comme dans [1], les modes étant découplés les uns des autres, nous omettons dans l'écriture des diverses grandeurs les indices $n l q$; nous adoptons aussi les conventions (5) :

$$
\mathfrak{H}_{1}=\mathfrak{H}_{z}, \mathfrak{H}_{2}=\mathfrak{H}_{\rho}-i \mathfrak{H}_{0}, \mathfrak{H}_{3}=\mathfrak{H}_{2}^{*} .
$$

Les équations (11), (12), (13) de [1] qui traduisent

$$
\begin{aligned}
\nabla \wedge \mathfrak{H} & =\mathfrak{J} \\
\nabla \cdot \mathfrak{H} & =0
\end{aligned}
$$

sont toujours valables à condition de remplacer $\mathfrak{J}$ par $\boldsymbol{S}+\mathcal{N}$. Elles s'écrivent :

$$
\begin{gathered}
\frac{\partial H_{1}}{\partial \rho}+\frac{l}{\rho} H_{1}-i q k H_{2}=-i\left(S_{2}+N_{2}\right) \\
-\frac{\partial H_{2}}{\partial \rho}+\frac{l-1}{\rho} H_{2}-i q k H_{1}=i\left(S_{1}+N_{1}\right) .
\end{gathered}
$$


De même, dans l'équation (15) relative aux courants induits dans le rotor, il faut remplacer $\mathfrak{J}$ par $\mathcal{N}$ :

$$
\frac{1}{\sigma} \nabla \wedge \mathcal{N}=-\mu_{0} \frac{\partial \mathscr{X}}{\partial t}+\mu_{0} \nabla \wedge(\mathbf{v} \wedge \mathscr{H})
$$

et l'on a de plus :

$$
\boldsymbol{\nabla} \cdot \mathcal{N}=-\nabla \cdot \boldsymbol{S} .
$$

On obtient ainsi les équations suivantes, comparables à (16) et (17) de [1] :

$$
\begin{gathered}
i \frac{\partial N_{1}}{\partial \rho}+i \frac{l}{\rho} N_{1}+q k N_{2}=-i \mu_{0} \sigma(n \omega+l \Omega) H_{2} \\
-i \frac{\partial N_{2}}{\partial \rho}+i \frac{l-1}{\rho} N_{2}+q k N_{1}=i \mu_{0}(n \omega+l \Omega) H_{1}+i \nabla \cdot \mathbf{S} \\
\nabla \cdot \mathbf{S}=\frac{1}{2} \frac{\partial}{\partial \rho}\left(S_{2}+S_{3}\right)-\frac{l-1}{2 \rho} S_{2}+\frac{l+1}{2 \rho} S_{3}+i q k S_{1} .
\end{gathered}
$$
le rotor

L'ensemble des équations (3), (6), (7) et (8) permet d'obtenir les équations du second ordre valables dans

$$
\begin{aligned}
& \frac{\partial^{2} H_{1}}{\partial \rho^{2}}+\frac{1}{\rho} \frac{\partial H_{1}}{\partial \rho}-\left[\frac{l^{2}}{\rho^{2}}+q^{2} k^{2}+i \mu_{0} \sigma(n \omega+l \Omega)\right] H_{1}=\frac{i}{2}\left(\frac{l-1}{\rho} S_{2}+\frac{l+1}{\rho} S_{3}-\frac{\partial S_{2}}{\partial \rho}+\frac{\partial S_{3}}{\partial \rho}\right) \\
& \frac{\partial^{2} H_{2}}{\partial \rho^{2}}+\frac{1}{\rho} \frac{\partial H_{2}}{\partial \rho}-\left[\frac{(l-1)^{2}}{\rho^{2}}+q^{2} k^{2}+i \mu_{0} \sigma(n \omega+l \Omega)\right] H_{2}=-i\left(\frac{l}{\rho} S_{1}+\frac{\partial S_{1}}{\partial \rho}\right)-q k S_{2} .
\end{aligned}
$$

Les équations dans le stator s'obtiennent en faisant, dans les deux expressions ci-dessus, $\sigma=0$ et $S=0$ (second membre nul). Elles sont donc très simples. Leur solution s'exprime immédiatement à l'aide des fonctions de Bessel [8], en posant toujours $a=i q k$ :

$$
\begin{aligned}
& H_{1}=G_{1} J_{l}(a \rho)+\widetilde{G}_{1} Y_{l}(a \rho) \\
& H_{2}=G_{2} J_{l-1}(a \rho)+\widetilde{G}_{2} Y_{l-1}(a \rho) \\
& H_{3}=G_{3} J_{l+1}(a \rho)+\widetilde{G}_{3} Y_{l+1}(a \rho) .
\end{aligned}
$$

La solution des équations (9) s'obtient en utilisant la méthode de la variation de la constante. On obtient finalement, en posant $b^{2}=-\left[q^{2} k^{2}+i \sigma \mu_{0}(n \omega+l \Omega)\right]$ et $Z=b \rho$ :

$$
\begin{aligned}
& H_{1}=\left(A_{1}+C_{1}\right) J_{l}(Z)+\left(\tilde{A}_{1}+\tilde{C}_{1}\right) Y_{l}(Z) \\
& H_{2}=\left(A_{2}+C_{2}\right) J_{l-1}(Z)+\left(\tilde{A}_{2}+\tilde{C}_{2}\right) Y_{l-1}(Z) \\
& H_{3}=\left(A_{3}+C_{3}\right) J_{l+1}(Z)+\left(\tilde{A}_{3}+\tilde{C}_{3}\right) Y_{l+1}(Z)
\end{aligned}
$$

avec :

$$
\begin{aligned}
& C_{1}=-\frac{i \pi}{4 b} \int_{0}^{b \rho}\left(S_{2} Y_{l-1}+S_{3} Y_{l+1}\right) Z \mathrm{~d} Z \\
& C_{2}=\frac{i \pi}{2 b} \int_{0}^{b \rho}\left(S_{1} Y_{l}-\frac{a}{b} S_{2} Y_{l-1}\right) Z \mathrm{~d} Z \\
& C_{3}=\frac{i \pi}{2 b} \int_{0}^{b \rho}\left(S_{1} Y_{l}+\frac{a}{b} S_{3} Y_{l+1}\right) Z \mathrm{~d} Z
\end{aligned}
$$

$\tilde{C}_{1}, \tilde{C}_{2}, \tilde{C}_{3}$ s'obtiennent par les mêmes expressions en changeant les $Y$ en $J$ ainsi que le signe de l'intégrale.
Les différentes constantes $A_{1}, \ldots, C_{1}, \ldots$ vont être déterminées à partir des conditions aux limites.

Au centre, pour $\rho=0$, le champ est fini. Les intégrales étant nulles pour $\rho=0$, cela donne $\widetilde{A}_{1}=\widetilde{A}_{2}$ $=\tilde{A}_{3}=0$.

En écrivant les relations du $1^{\mathrm{er}}$ ordre, telles que (3), hors du rotor, on trouve encore :

$$
\begin{aligned}
& G_{1}=G_{2}=-G_{3} \\
& \tilde{G}_{1}=\tilde{G}_{2}=-\tilde{G}_{3} .
\end{aligned}
$$

On montre aussi que l'on a toujours, comme dans [1]

$$
2 a A_{1}-b A_{2}+b A_{3}=0 \text {. }
$$

Il reste donc à écrire la continuité des champs à la surface du rotor et le fait que le champ $H$ est normal à la ceinture. Cette dernière condition est satisfaite dès que $H_{1}(a F)=0$ (cf. [1]). Les champs créés par les courants sources $\boldsymbol{S}$ s'écrivent donc :

- dans le rotor

$$
\begin{aligned}
& H_{1}=\left(A_{1}+C_{1}\right) J_{l}(b \rho)+\tilde{C}_{1} Y_{l}(b \rho) \\
& H_{2}=\left(A_{2}+C_{2}\right) J_{l-1}(b \rho)+\tilde{C}_{2} Y_{l-1}(b \rho) \\
& H_{3}=\left(A_{3}+C_{3}\right) J_{l+1}(b \rho)+\tilde{C}_{3} Y_{l+1}(b \rho)
\end{aligned}
$$

- hors rotor

$$
\begin{aligned}
& H_{1}=G_{1} J_{l}(a \rho)+\tilde{G}_{1} Y_{l}(a \rho) \\
& H_{2}=G_{1} J_{l-1}(a \rho)+\tilde{G}_{1} Y_{l-1}(a \rho) \\
& H_{3}=-G_{1} J_{l+1}(a \rho)-\widetilde{G}_{1} Y_{l+1}(a \rho) .
\end{aligned}
$$


Les cinq constantes $A_{1}, A_{2}, A_{3}, G_{1}, \widetilde{G}_{1}$ s'obtiennent en résolvant le système linéaire :

$$
\begin{aligned}
& 2 a A_{1}-b A_{2}+b A_{3}=0 \\
& A_{1} J_{l}(b R)-G_{1} J_{l}(a R)-\tilde{G}_{1} Y_{l}(a R)=-C_{1} J_{l}(b R)-\widetilde{C}_{1} Y_{l}(b R) \\
& A_{2} J_{l-1}(b R)-G_{1} J_{l-1}(a R)-\widetilde{G}_{1} Y_{l-1}(a R)=-C_{2} J_{l-1}(b R)-\widetilde{C}_{2} Y_{l-1}(b R) \\
& A_{3} J_{l+1}(b R)+G_{1} J_{l+1}(a R)+\widetilde{G}_{1} Y_{l+1}(a R)=-C_{3} J_{l+1}(b R)-\tilde{C}_{3} Y_{l+1}(b R) \\
& G_{1} J_{l}(a F)+\tilde{G}_{1} Y_{l}(a F)=0
\end{aligned}
$$

où les intégrales $C_{1}, \ldots, \tilde{C}_{1}, \ldots$ sont calculées pour $\rho=R$. Si la machine n'est pas entourée d'une ceinture, la dernière ligne de ce système est remplacée par :

$$
G_{1}+i \widetilde{G}_{1}=0 \text {. }
$$

\section{Détermination des courants sources.}

Il nous faut tout d'abord calculer les courants induits dans le rotor infini et séparer ensuite ceux qui sont dans les «bouts". Si l'on se reporte à l'équation (3), on y voit que l'expression du courant induit $N$ se déduit immédiatement de celle de $H$ (puisque $S$ est connu). On en déduit, en se reportant aux expressions des champs (13) et en utilisant les relations entre fonctions de Bessel et leurs dérivées [8] :

$$
\begin{aligned}
& N_{1}=i\left[a\left(A_{1}+C_{1}\right)-b\left(A_{2}+C_{2}\right)\right] J_{l}(b \rho)+i\left(a \tilde{C}_{1}-b \tilde{C}_{2}\right) Y_{l}(b \rho) \\
& N_{2}=i\left[-a\left(A_{1}+C_{2}\right)+b\left(A_{1}+C_{1}\right)\right] J_{l-1}(b \rho)+i\left(-a \widetilde{C}_{2}+b \tilde{C}_{1}\right) Y_{l-1}(b \rho) \\
& N_{3}=i\left[a\left(A_{3}+C_{3}\right)+b\left(A_{1}+C_{1}\right)\right] J_{l+1}(b \rho)+i\left(a \tilde{C}_{3}+b \tilde{C}_{1}\right) Y_{l+1}(b \rho) .
\end{aligned}
$$

Lors de l'itération d'ordre 0 , c'est-à-dire lors du calcul de l'action des seuls courants statoriques, les trois expressions ci-dessous sont encore valables avec $C_{1}=\cdots=\tilde{C}_{1}=\cdots=0$ et elles donnent $I_{\mathrm{i}}$.

Ayant ainsi les courants induits $\mathcal{N}\left(\right.$ ou $\mathfrak{J}_{\mathrm{i}}$ ), on peut en déduire facilement les courants sources $\mathbf{S}^{\prime}$ (ou \$) qui seront utilisés dans l'itération suivante. Ils sont tels que :

$$
\boldsymbol{\delta}^{\prime}=\Gamma \boldsymbol{N}
$$

où $\Gamma$.est défini pour $\rho<R$ par :

$$
\Gamma(\rho, \theta, z)=0 \quad \text { si } \quad z_{A}<z<z_{B} \quad \text { et } \quad z_{C}<z<z_{D}
$$

$\Gamma(\rho, \theta, z)=-1$ ailleurs .

Autrement dit $I$ est nul dans le rotor, égal à -1 à l'extérieur.

Il est facile de déterminer les coefficients de Fourier de $\Gamma$. Comme il est indépendant de $\theta$ et $z$, on a :

$$
\Gamma=\sum_{q=\mathbf{Z}} \gamma_{q} \mathrm{e}^{i q k z}
$$

avec

$$
\begin{aligned}
\gamma_{2 q+1} & =0 \\
\gamma_{2 q} & =\frac{(-1)^{q}}{\pi q} \sin q k H_{R} \\
\gamma_{0} & =-1+\frac{k H_{R}}{\pi} .
\end{aligned}
$$

On en déduit :

$$
\mathbf{S}^{\prime}=\sum \mathbf{S}_{n l q}^{\prime} \mathrm{e}^{i(n \omega t+l \theta+q k z)}
$$

avec

$$
\mathbf{S}_{n l q}^{\prime}=\sum_{q^{\prime}} \gamma_{q-q^{\prime}} \mathbf{N}_{n l q^{\prime}}
$$

On appliquera tout d'abord cette formule avec le courant induit $I_{\mathrm{i}, n l q^{\prime}}$ pour en déduire $S_{n l q}$. Or, on sait que $q^{\prime}$ est forcément impair (cf. (74) de [1]; cette propriété y est établie dans un cas particulier, mais on peut montrer qu'elle est générale suite au choix d'un système comportant des machines contiguës symétriques). $q-q^{\prime}$ est pair d'après (21). On en déduit que $q$ est impair, ce qui était prévisible car les symétries de $\boldsymbol{S}$ sont les mêmes que celles de $\mathfrak{J}_{\mathbf{i}}$ (et $\mathfrak{J})$.

\section{Calcul numérique.}

a) On reprend le programme établi en [1] que l'on complète par le calcul des courants dans le rotor (expression (17) avec tous les $C$ nuls).

b) On applique (22) qui donne les courants sources. On calcule les diverses intégrales $C$ par (12). On résout le système (15).

On en déduit les champs (13) et (14). En ajoutant (13) aux champs calculés dans l'itération précédente, on détermine alors, comme précédemment, le couple par intégration sur la surface du rotor. Les expres- 
sions (14) nous permettent de calculer, par intégration sur le volume du bobinage, l'écart de tension qui apparaît et par suite les écarts qui en résultent sur les puissances actives et réactives. Si les écarts relatifs sont très faibles, on arrête le calcul. Sinon, on calcule les courants induits (17) et l'on reprend en $b$ ).

\section{Résultats.}

Nous avons considéré une machine avec ceinture dont le rotor a $25 \mathrm{~cm}$ de diamètre et dont le bobinage est de structure hélicoïdale (cf. [1]). Nous avons alors calculé ses performances pour diverses longueurs du bobinage en considérant à chaque fois le cas où le rotor est très long et celui où il a la même longueur que le bobinage (rappelons que, dans cette modélisation, la longueur de la ceinture est supposée infinie). Les figures 3 rapportent les résultats obtenus; les figures 4 les précisent pour une machine "carrée " (longueur égale au diamètre).

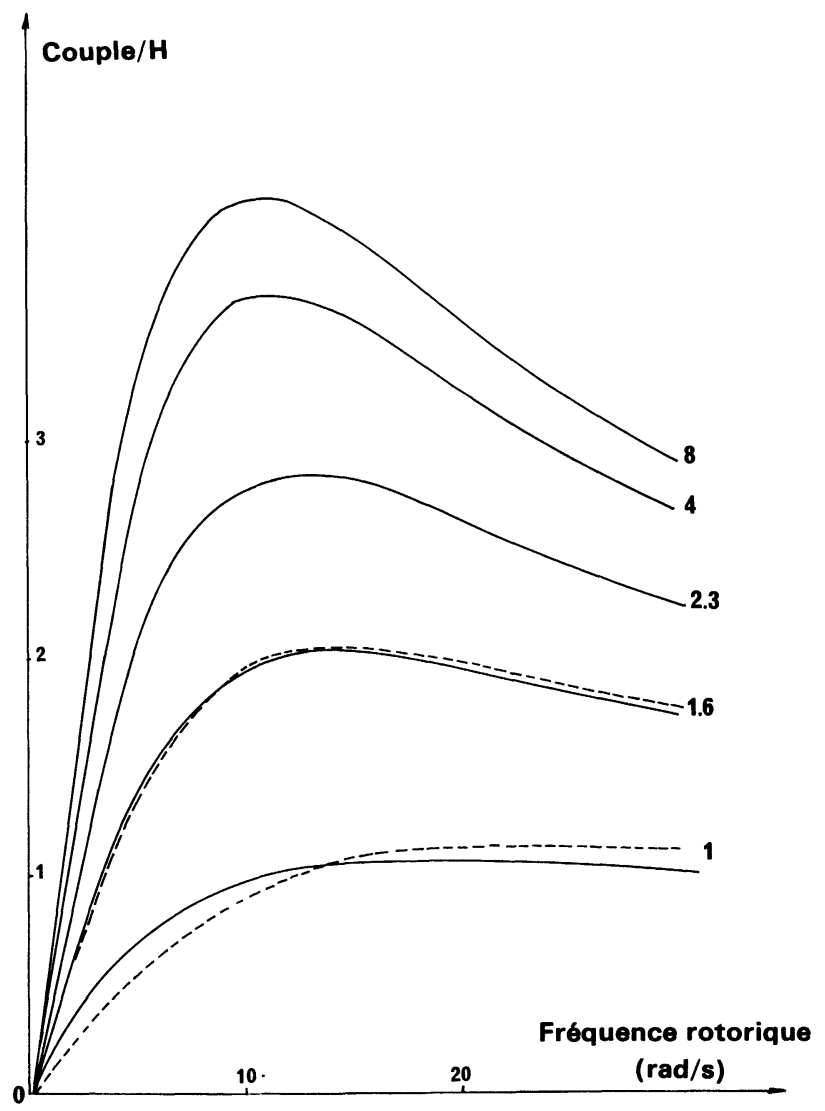

(a)

Fig. 3. - Couple par unité de longueur (a), puissance réactive par unité de longueur (b) et facteur de puissance (c) pour diverses machines ayant des longueurs différentes, mais les autres dimensions identiques. Les courbes en traits pleins correspondent au rotor très long et celles en pointillés au rotor de même longueur que le bobinage. Les unités de couple et puissance sont arbitraires mais sont les mêmes pour toutes les figures.

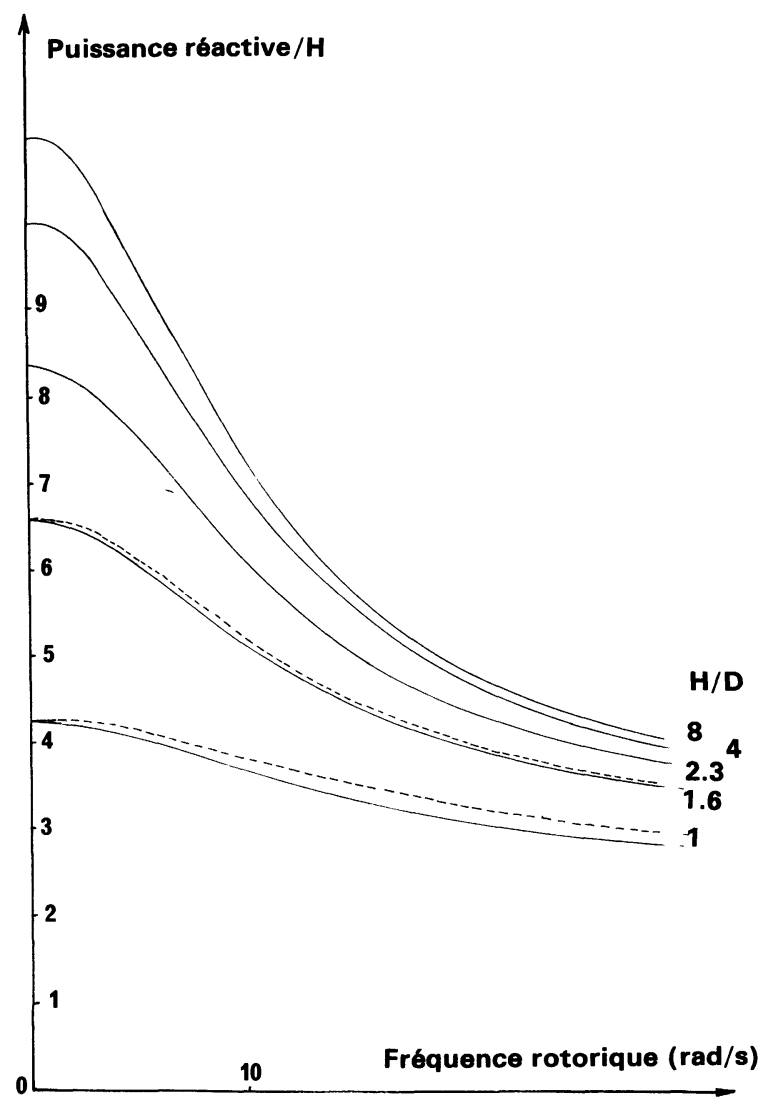

(b)

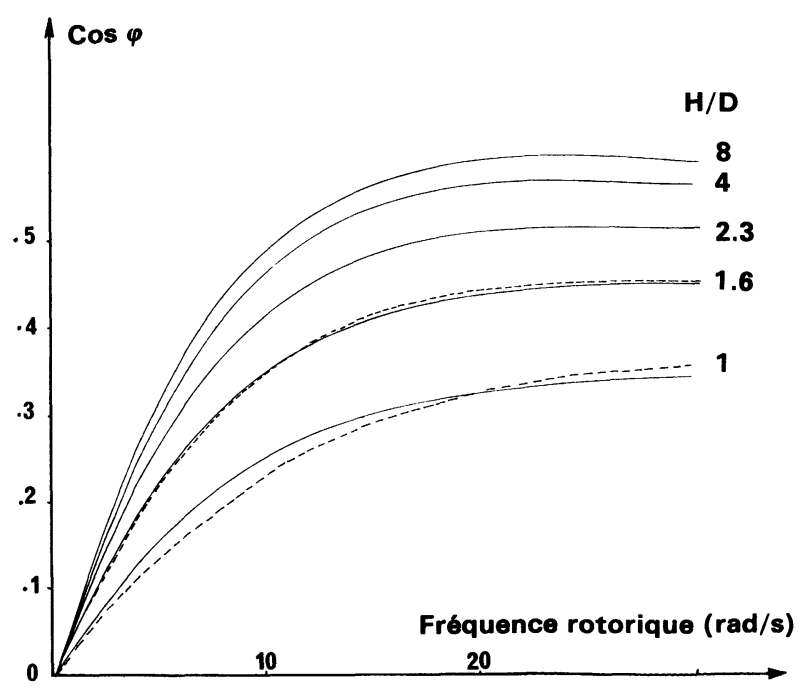

(c)

[Torque per unit length (a), reactive power per unit length (b) and power factor (c) for machines having different lengths and equal other dimensions. The continuous curves are for a very long rotor and the dotted curves for a rotor having the same length as the winding. The torque and power units are arbitrary but are the same for all the curves.] 


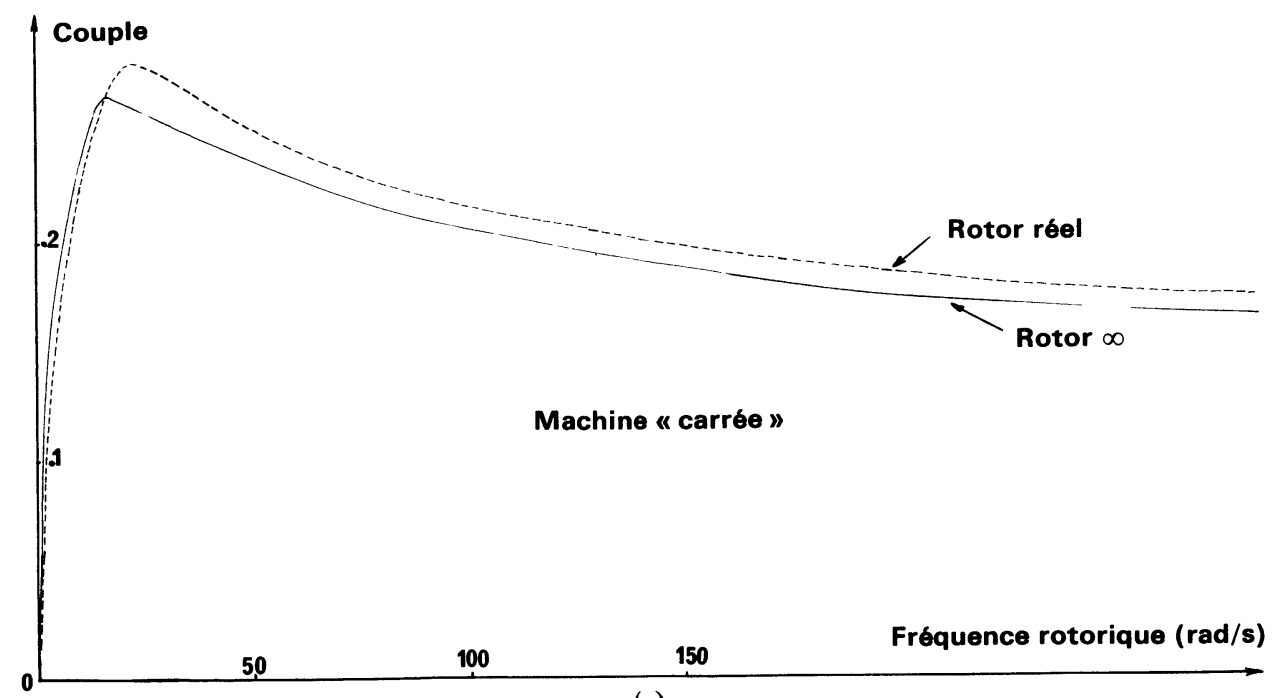

(a)

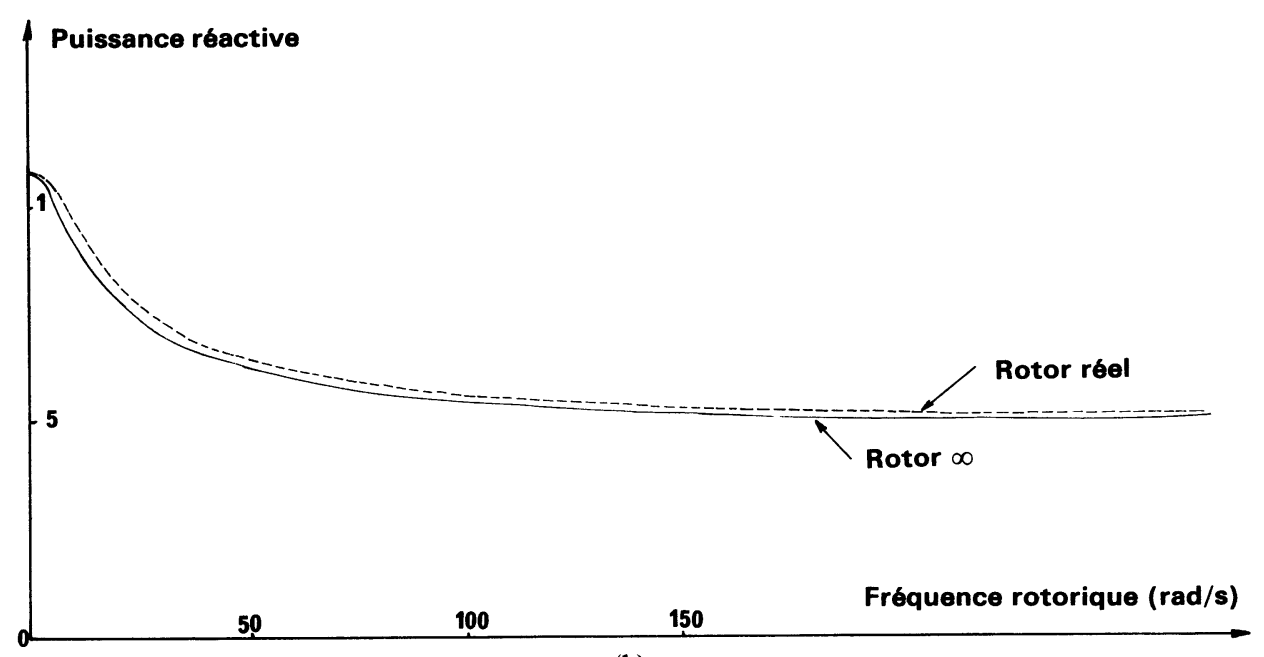

(b)

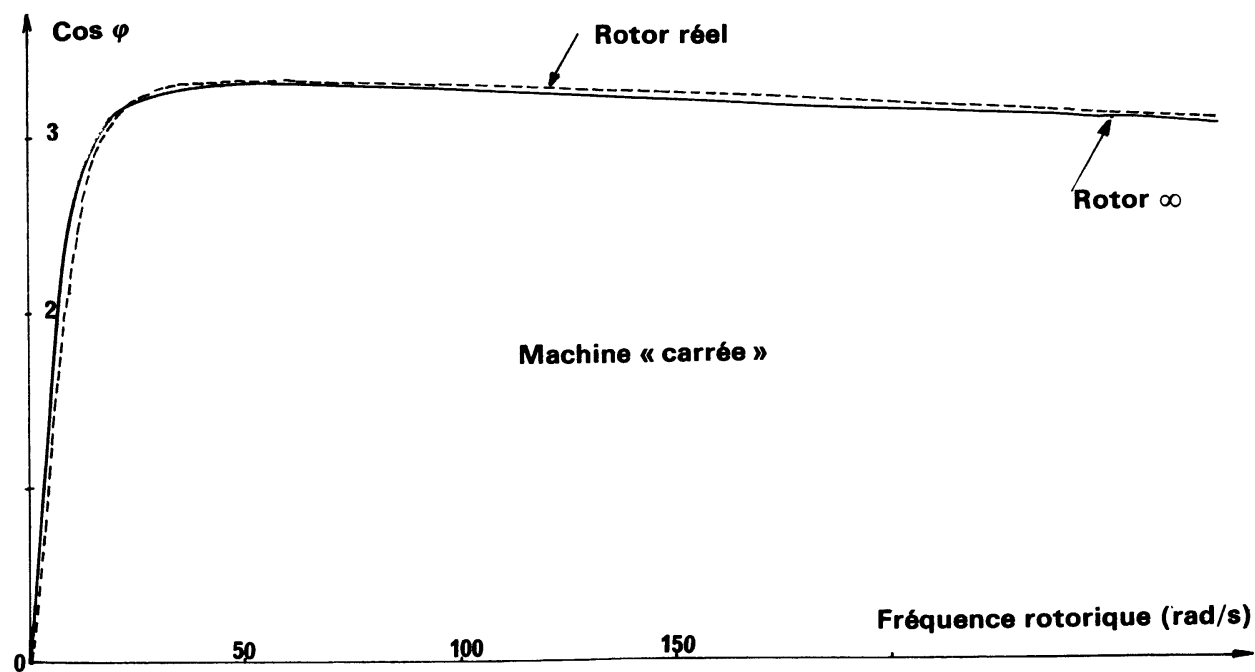

(c)

Fig. 4. - Couple (a), puissance réactive (b) et facteur de puissance (c) pour une machine " carrée » (longueur du bobinage égale au diamètre du rotor). La courbe en traits pleins correspond au rotor très long, celle en pointillés est obtenue avec 9 itérations.

[Torque (a), reactive power (b) and power factor (c) for a « square » machine (winding length equal to rotor diameter). The continuous curve corresponds to a very long rotor, the dotted is obtained with 9 iterations.] 
Nous retrouvons que les performances d'une machine se détériorent lorsque la longueur du bobinage décroît (le couple par unité de longueur et le $\cos \varphi$ diminuent; le couple maximal est obtenu pour une fréquence rotorique plus grande donc pour des pertes rotoriques plus fortes). Comme prévisible, les corrections apportées par les itérations successives au calcul du " rotor infini " sont négligeables lorsque le bobinage et par suite le rotor réel sont très longs ; elles augmentent lorsque celui-ci se raccourcit. Lorsque le rotor a pour longueur quelques diamètres, les effets sont très faibles (quelques $\%$ ); une seule itération suffit. Lorsque sa longueur devient égale à 1,6 diamètre, il est bon d'effectuer deux itérations, mais l'approximation du rotor infini est encore très bonne (Fig. 5). Pour la machine « carrée » (longueur égale au diamètre), il est nécessaire d'effectuer 5 ou 6 itérations (les Figs. 6 comparent les résultats obtenus avec 4 et 9 itérations).

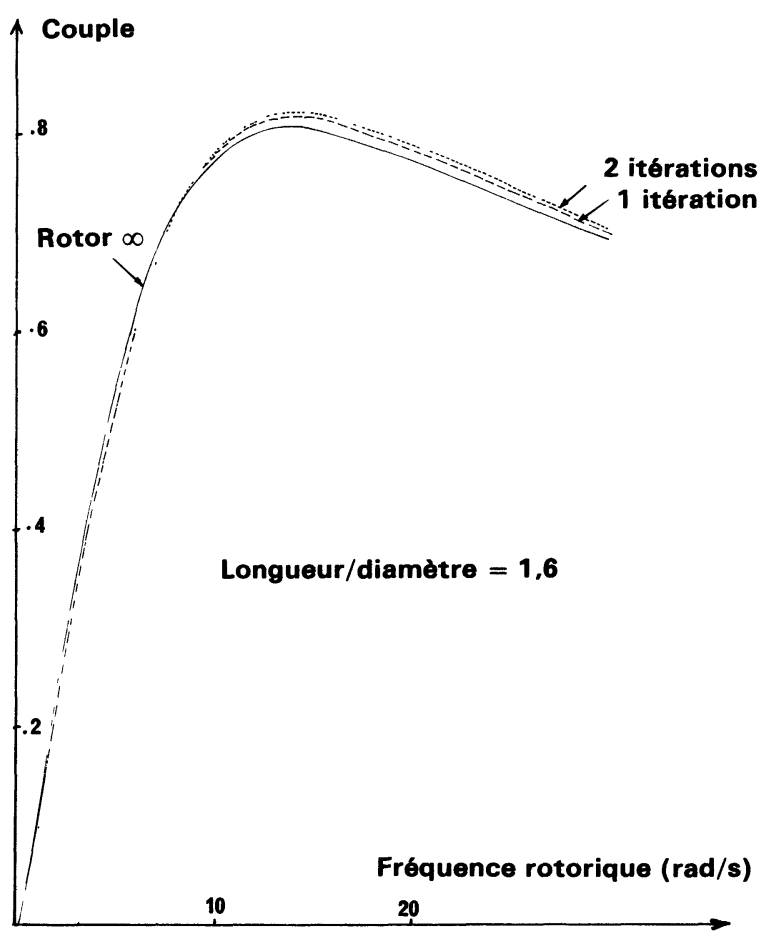

Fig. 5. - Couple lorsque longueur du bobinage/diamètre $=1,6$.

[Torque with winding length/rotor diameter $=1.6$.]

Le résultat relatif aux rotors longs est bien en accord avec les expériences que nous avons déjà effectuées et qui concernaient d'une part un système où le rapport longueur rotor/diamètre était égal à 3,5 [6] et un prototype où ce même rapport valait 6,25 [2]. Dans les deux cas, nous avions constaté que la modélisation semi-tridimensionnelle à rotor long donnait une description tout à fait correcte.

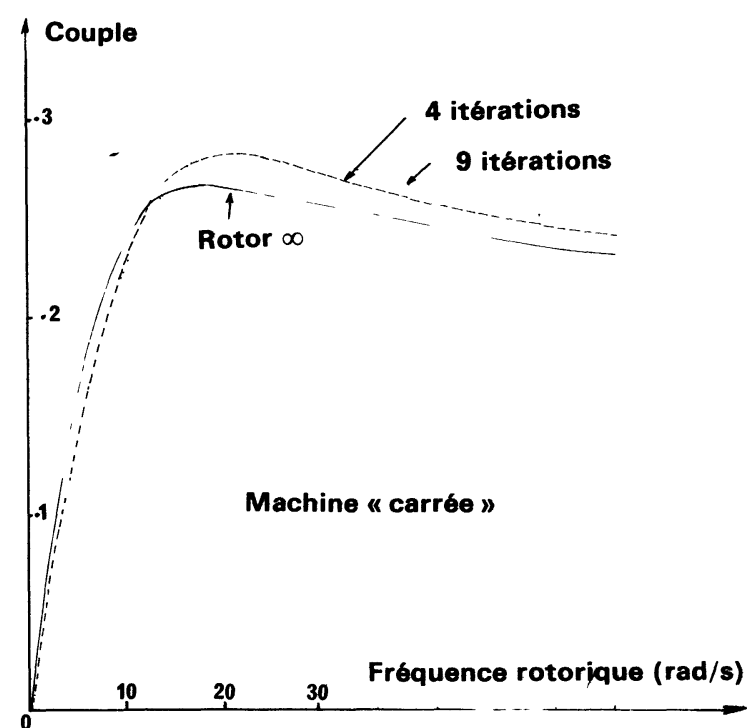

(a)

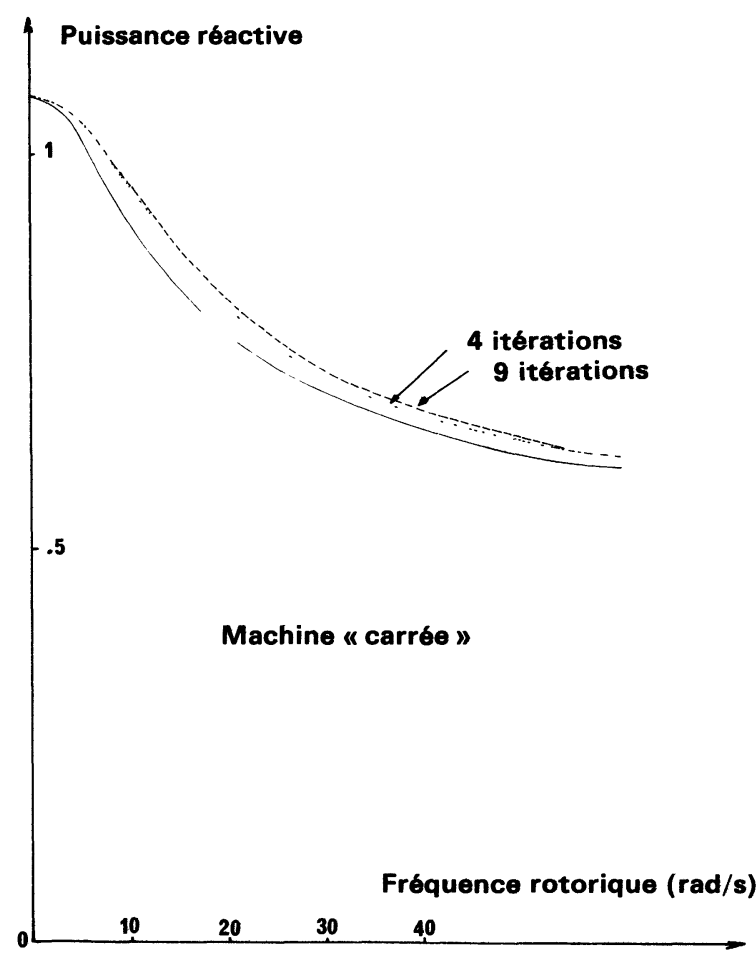

(b)

Fig. 6. - Influence du nombre d'itérations lors du calcul de la machine « carrée ».

[Influence of the number of iterations in the calculus of the " square " machine.]

De façon générale, on constate que le fait que le rotor ait la même longueur que le bobinage plutôt qu'une longueur très supérieure entraîne :

- une diminution du couple et une diminution $\mathrm{du}\langle\cos \varphi »$ pour les fréquences rotoriques faibles (inférieures à celle donnant le couple maximal); 
- une augmentation du couple et une augmentation $\mathrm{du}$ « $\cos \varphi »$ pour les fréquences rotoriques élevées ;

- un déplacement du maximum du couple vers des fréquences rotoriques plus grandes;
- une augmentation de la puissance réactive.

Signalons que le facteur de puissance est calculé en ignorant les pertes joules dans le stator; leur prise en compte augmenterait un peu le « $\cos \varphi »$, et donnerait la valeur effective dans une utilisation.

\section{Bibliographie}

[1] Rioux-Damidau, F., Revue Phys. Appl. 18 (1983) 113.

[2] Rioux, C., Bleiss, C. A., Rioux-Damidau, F., SultaNEM, F., RGE 10 (1984) 619.

[3] Rioux, C., Sultanem, F., J. Appl. Phys. 50 (1979) 574.

[4] Bleiss, C. A., Demonchy, M., Rioux, C., Electr. Machines Electromech. 7 (1982) 351.

[5] Letellier, J. L., Thèse de Docteur-Ingénieur, Orsay (1983).
[6] Bleiss, C. A., Letellier, J. L., Rioux, C., RiouxDamidau, F., Revue Phys. Appl. (soumis).

[7] Bossavit, A., Vérité, J. C., IEEE Trans. on Magn. MAG 19, 6 (1983) 2665.

[8] Abramovitz, M., Stegun, J., Handbook of Mathematical functions (Dover Publications, Inc., New York) 1965. 